\title{
A baixa duração do sono está associada com a inatividade física em adolescentes Amazonenses?
}

\author{
The short sleep duration associated with physical inactivity among \\ Amazonas adolescents?
}

Geraldo Jose Ferrari Junior'; André de Araújo Pinto'; Andreia Pelegrini'; Rita Maria dos Santos Puga Barbosa²; Marcus Vinicius Nahas³; Érico Gomes Pereira Felden?

\begin{abstract}
Resumo
Este estudo teve como objetivo analisar os fatores associados à baixa duração do sono em adolescentes Amazonenses. Trata-se de um estudo epidemiológico transversal, conduzido com 2517 adolescentes (1106 do sexo masculino e 1411 do sexo feminino), com idades de 14 a 19 anos, matriculados em escolas públicas de ensino médio no estado do Amazonas. Além da baixa duração do sono (< oito horas/dia) foram investigadas informações sobre sexo, faixa etária, ano escolar, turno escolar, renda familiar, situação ocupacional e atividade física. Associações entre a baixa duração de sono com as demais variáveis foram analisadas por meio da regressão de Poisson. A prevalência de baixa duração de sono foi de (51,2\%; IC95\%: 49,2-52,9). Adolescentes mais velhos de 18 a 19 anos ( $\mathrm{RP}=1,14$; IC95\%: 1,01-1,29), que estudam no turno matutino $(\mathrm{RP}=1,37$; IC95\%: $1,25-1,48$ ) e noturno (RP= 1,16; IC95\%: 1,04-1,28), os que trabalham $(\mathrm{RP}=1,34$; IC95\%: $1,23-1,45)$ e são inativos fisicamente ( $\mathrm{RP}=1,27$; IC95\%: 1,06-1,53) apresentaram maior risco para a baixa duração do sono. Os resultados mostram que fatores biológicos, comportamentais e ambientais são fatores importantes para a duração do sono dos adolescentes.
\end{abstract}

\section{Palavras-chave}

Atividade Motora; Saúde do adolescente; Sono.

\begin{abstract}
This study aimed to analyze the factors associated with short sleep duration in adolescents from Amazonas. It is a cross-sectional epidemiological study conducted with 2,517 adolescents (1,106 males and 1,411 females), aged 14 to 19 years, enrolled in public schools in the state of Amazonas. In addition to the short sleep duration (< eight hours per day), information about sex, age group, school grade, turn school, family income, occupation and physical activity were investigate. Associations between short sleep duration with the other variables were analyzed using Poisson regression. Prevalence of short sleep duration found in adolescents Amazonas was (51.2\%; 95\% CI: 49.2-52.9). Oldest adolescents from 18 to 19 years ( $P R=$ 1.14; 95\% CI: 1.01-1.29), who are studying in the morning turn $(P R=1.37 ; 95 \%$ CI: $1.25-1.48)$ and nocturnal $(P R=1.16 ; 95 \%$ CI: 1.04-1.28), those who work $(P R=1.34 ; 95 \% C I: 1.23-1.45)$ and are physically inactive $(R P=1.27 ; 95 \% C I=1.06-1.53)$ presented bigher risk for short sleep duration. The results show that biological, behavioral and environmental factors are important during period of adolescents' sleep.
\end{abstract}

\section{Keywords}

Motor Activity, Adolescent Health, Sleep.

\section{Introdução}

A adolescência é marcada por alterações de hábitos, destacando-se a redução da atividade física $(\mathrm{AF})^{1,2}$, inserção no mundo do trabalho ${ }^{3}$ e a privação do sono (dormir menos do que a autopercepção de necessidade de sono $)^{4}$. A privação do sono, especialmente,

1 Programa de Pós-Graduação em Ciências do Movimento Humano, Centro de Ciências da Saúde e do Esporte, Universidade do Estado de Santa Catarina, Florianópolis, SC, Brasil.

2 Programa de Pós-Graduação em Sociedade e Cultura na Amazônia, Faculdade de Educação Física e Fisioterapia, Universidade Federal do Amazonas, Manaus, AM, Brasil

3 Programa de Pós-Graduação em Educação Física, Centro de Desportos, Universidade Federal de Santa Catarina, Florianópolis, SC, Brasil. está associada ao atraso no ciclo vigília/sono o qual é gerado pela relação de fatores intrínsecos e extrín$\operatorname{secos}^{5}$. Os fatores intrínsecos, que geram o atraso da fase de sono, estão ligados às alterações nos processos de regulação do sono, principalmente ao Processo Homeostático que, se caracteriza por um acúmulo de propensão ao sono mais lento durante o dia. Este atraso de fase é mais acentuado no final da adolescência ${ }^{6}$. Já os fatores extrínsecos dizem respeito aos compromissos sociais, ocupacionais e escolares ${ }^{5}$.

Observa-se que alguns fatores associados à baixa 
duração do sono de adolescentes são os turnos de estudo matutino e noturno, ser mais velho ${ }^{4,7}$, ter qualidade do sono ruim $^{7}$, possuir ocupação, também como, trabalhar e ainda estudar de tarde ${ }^{8}$, ser de classe social alta e o hábito de dormir durante o dia ${ }^{9}$. Esses fatores têm sido associados à elevada prevalência de baixa duração do sono que, em alguns estudos, apresentam percentuais acima de $50 \%{ }^{4,8,10}$ e oscilam de acordo com o contexto socioeconômico e geográfico de onde são realizados ${ }^{11}$.

$\mathrm{O}$ ato de dormir pouco está associado com inúmeros riscos à saúde ${ }^{12}$, pois o sono, por meio de seus efeitos fisiológicos, proporciona a termorregulação corporal, promove o restabelecimento das condições energéticas e ajuda na restauração dos sistemas corporais degastados durante a vigília, funções estas que podem ser prejudicadas pela baixa duração do sono ${ }^{13}$. Ademais, a privação do sono também está associada a problemas com a aprendizagem como o baixo rendimento escolar ${ }^{11}$, que pode ser reflexo de dificuldades de concentração e memorização de novas informações ocasionadas pela sonolência diurna proveniente da baixa duração do sono ${ }^{14}$.

Dentre os fatores comportamentais que podem contribuir para o alcance de horas recomendadas de sono destaca-se a prática regular de $\mathrm{AF}$, que ajuda no aumento da duração, na diminuição da latência e melhora a eficiência do sono ${ }^{15}$. Apesar dos possíveis efeitos da AF sobre o sono, sua prática não tem sido incorporada com frequência pelos adolescentes, os quais vêm frequentemente adotando um estilo de vida hipocinético ${ }^{1,2}$. Isso sugere que adolescentes com baixos níveis de AF além de estarem em risco de desenvolver problemas de saúde inerentes, podem ter o risco aumentado se esses desfechos (AF e sono) estiverem relacionados ${ }^{15}$.

Diante da importância da AF para o sono dos adolescentes e, ao considerar que as prevalências de baixa duração de sono variam de acordo com contexto geográfico ${ }^{11}$, faz-se necessário a condução de estudos no Brasil que, em função de suas dimensões continentais, apresenta regiões com diferentes características comportamentais sendo, portanto, impossível fazer generalizações sobre a associação entre AF e sono. Na região Norte, especialmente no Amazonas, a carência de estudos sobre a AF e principalmente o sono em adolescentes é evidente, o que certamente é reflexo da ausência de programas de pós-graduação em educação física e de grupos de estudos na área que limita uma visão ampliada da temática no Brasil.

A relevância desses estudos se destaca por orientar a elaboração de políticas públicas que favoreçam a prática de $\mathrm{AF}$ regular e melhora dos padrões de sono nos adolescentes, evitando-se maiores onerações com a saúde e educação que podem ser provenientes da inatividade física e baixa duração do sono durante a adolescência. Diante de tais considerações, o presente estudo teve como objetivo analisar os fatores associados à baixa duração do sono em adolescentes Amazonenses.

\section{Métodos}

Esse estudo observacional transversal faz parte de uma pesquisa de abrangência maior intitulada "Estilo de Vida e Indicadores de Saúde de Escolares do Ensino Médio do Amazonas" realizada em 2011. A população alvo foi de adolescentes, de ambos os sexos, com idade de 14 a 19 anos, matriculados nos três anos do ensino médio em escolas públicas estaduais de cinco cidades do Amazonas (São Gabriel da Cachoeira, Presidente Figueiredo, Parintins, Manaus e Itacoatiara). A escolha das cidades deu-se de forma intencional, em virtude da localização geográfica das mesmas que são ribeirinhas, sendo mais acessível chegar até elas em relação as demais cidades do Amazonas.

Segundo as informações da Secretária de Educação do Estado o percentual de adolescentes matriculados no ensino médio era de, aproximadamente 43,0\% 
e 88.560 adolescentes encontravam-se matriculados no ensino médio nas cinco cidades no ano em que o estudo foi realizado. A partir desse quantitativo, foi estabelecido o processo de seleção amostral. Na cidade de Manaus a seleção amostral ocorreu da seguinte forma: a) proporcional por distritos de educação (n=6); b) estratificado por escolas públicas estaduais, observando-se o volume (grande porte: 500 alunos ou mais; médio porte: de 201 a 499 alunos; e pequeno porte: até 200 alunos); e c) por conglomerado de turnos, turmas e ano escolar, em que todos os adolescentes presentes em sala no momento da coleta foram convidados a participar do estudo. Em São Gabriel da Cachoeira, Parintins e Presidente Figueiredo, considerando o baixo número de escolas, empregou-se os estágios "b" e "c". $\mathrm{Na}$ cidade de Presidente Figueiredo foi realizado um censo nas duas únicas escolas, e todos os adolescentes foram incluídos no estudo.

O tamanho da amostra foi determinado considerando os múltiplos comportamentos investigados, adotando-se prevalência estimada de 50\% (desfecho desconhecido), nível de confiança de $95 \%$, erro de cinco pontos percentuais e efeito de delineamento de 1,5 por se tratar de um processo amostral por conglomerado ${ }^{16}$. Fora acrescido $10 \%$ no tamanho da amostra para reduzir a ocorrência de possíveis perdas/recusas, chegando ao tamanho da amostra em cada uma das cidades (São Gabriel da Cachoeira $=423$; Presidente Figueiredo= 264; Parintins $=587$; Itacoatia$\mathrm{ra}=580$; Manaus $=631$ ), um total de 2.485 adolescentes, sendo que o questionário foi aplicado a 3.267 adolescentes. Para o estudo dos fatores associados o tamanho da amostra calculado possibilitou detectar razões de prevalência de 1,11 ou mais para expostos, com poder de 80\% e nível de confiança de 95\%. Dos 3.267 questionários recolhidos 382 foram considerados perdas (em virtude de acidentes/transtornos sucedidos durante a logística entre as cidades) e 368 foram excluídos (por não contemplarem a faixa etária estabelecida para o estudo).

\section{Procedimentos e avaliações}

A aprovação do estudo deu-se por meio do Comitê de Ética em Pesquisa com Seres Humano da Universidade Federal do Amazonas (CAAE-0302.0.115.000-11), de acordo com a Declaração de Helsinki, resolução n 196/96. A coleta de dados ocorreu durante as aulas teóricas de Educação Físicas, em dias e horários estabelecidos previamente, em acordo com os responsáveis das escolas. Em um primeiro encontro, os adolescentes foram informados sobre a importância da pesquisa e receberam o Termo de Consentimento Livre e Esclarecido-TCLE para que fosse assinado por seus pais/responsáveis, autorizando a participação de seu filho na pesquisa. No segundo encontro, um questionário autopreenchível foi aplicado por uma equipe de pesquisadores treinados, designada à realização da coleta de dados. Somente foram incluídos, os adolescentes que entregaram o TCLE assinado por seus pais/responsáveis e aqueles que assinaram o Termo de Assentimento.

Os dados foram coletados mediante a utilização do questionário COMPAC II (Estilo de Vida de Adolescentes Catarinenses) ${ }^{17}$ que foi desenvolvido a partir de instrumentos já validados. A duração do sono (variável dependente) foi operacionalizada por meio da seguinte pergunta: "Em média, quantas horas você dorme por dia em uma semana normal". A escala de resposta continha sete itens a) menos de seis horas; b) seis horas; c) sete horas; d) oito horas; e) nove horas; f) 10 horas e g) mais de 10 horas. Os adolescentes com tempo inferior a oito horas de cama foram considerados com baixa duração, ponto de corte estipulado pela National Sleep Foundation $^{18}$, que reuniu vários especialistas sobre sono.

As variáveis exploratórias foram sexo (masculino, feminino), faixa etária (14-15, 
16-17, 18-19 anos), turno escolar (matutino, vespertino, noturno), situação ocupacional (trabalha, não trabalha) e renda mensal familiar, a qual considerou o salário em vigor no ano de 2011 (R\$ 545,00). A informação sobre a renda foi obtida por meio da seguinte questão: "Qual a renda mensal da sua familia?", tendo como alternativas de respostas a) atédois salários; b) de três a cinco salários; c) de seis a 10 salários ou mais.

O nível de AF foi estimado por meio de duas questões sendo que a primeira diz respeito a frequência - "Durante uma semana normal (típica), em quantos dias você pratica atividades físicas moderadas a vigorosas (atividade física no lazer, no trabalho e no deslocamento)?", como respostas os adolescentes tinham oito alternativas que variou de 0 a sete dias. A segunda questão referia-se a duração - "Durante uma semana normal (típica), quanto tempo você pratica atividades físicas moderadas a vigorosas (atividade física no lazer, no trabalho e no deslocamento)?”, como respostas os adolescentes tinham quatro alternativas a) não pratico; b) menos de 30 minutos por dia; c) 30 a 59 minutos por dia e d) 60 minutos ou mais por dia. Essas questões foram previamente testadas em 107 adolescentes com idade de 15 a 19 anos da cidade Florianópolis, apresentando-se concordância de 92,8\% e índice kappa igual a 0,81 para AF moderada a vigorosa ( $<300$ minutos por semana $v s . ~ \geq 300$ minutos por semana) (dados não publicados).

Os adolescentes que relataram não praticar AF foram considerados inativos, os que realizaram alguma $\mathrm{AF}$, mas que não atingiram 60 minutos diários insuficientemente ativos, e os que realizaram 60 minutos ou mais em todos os dias da semana foram considerados ativos fisicamente ${ }^{19}$.

\section{Análise estatística}

Os dados foram analisados utilizando-se a estatística descritiva (distribuição de frequências) e inferencial. A diferença de proporções de baixa duração do sono entre os sexos foi verificada por meio da sobreposição dos intervalos de confiança de $95 \%$. Como não houve diferenças entre as proporções as análises de associação foram verificadas sem estratificação por sexo. A associação entre as variáveis foi analisada utilizando-se a regressão de Poisson (bruta e ajustada) com estimativa robusta estimando-se a razão de prevalência (RP) e os respectivos intervalos de confiança de $95 \%$. Na análise ajustada todas as variáveis foram incluídas no modelo independentemente do valor de $\mathrm{p}$ na análise bruta. Os dados foram administrados no Software Statistical Package for Social Science - (SPSS, versão 20.0) e o nível de significância adotado foi de 5\%.

\section{Resultados}

Dos 3.267 questionários recolhidos, 11,69\% dos dados foram considerados perdas perdidos (em virtude de acidentes/transtornos sucedidos durante a coleta) e 11,26\% foram excluídos (por não contemplarem a faixa etária estabelecida para o estudo), resultando em uma amostra total de 2.517 adolescentes. Dentre os adolescentes investigados, a maioria era do sexo feminino (56,1\%). A maior parte dos adolescentes tinha idade de 16 a 17 anos (56,1\%), estudava no turno vespertino (52,3\%), era de famílias com renda mensal de até dois salários (64,0\%), não trabalhava (81,9\%). A maioria dos adolescentes foi classificada como sendo insuficientemente ativa $(71,7 \%)$ (Tabela 1$)$.

A prevalência de baixa duração do sono nos adolescentes (51,2\%; IC95\%: 49,3$52,8)$ foi apresentada na Figura 1. Não foi verificada diferença entre as prevalências de baixa duração do sono entre o sexo masculino (52,9\%; IC95\%: 51,0-54,6) e feminino (49,8\%; IC95\%: 47,8-51,5). 
TABELA 1 - Características gerais dos adolescentes de escolas públicas de ensino médio, na amostra total e de acordo com o sexo. Amazonas, 2011 ( $n=2.517)$.

\begin{tabular}{|c|c|c|c|}
\hline Variáveis & $\begin{array}{l}\text { Total } \\
\text { n (\%) }\end{array}$ & $\begin{array}{c}\text { Masculino } \\
\text { n (\%) }\end{array}$ & $\begin{array}{c}\text { Feminino } \\
\mathrm{n}(\%)\end{array}$ \\
\hline \multicolumn{4}{|l|}{ Faixa etária (anos) } \\
\hline $14-15$ & $519(20,6)$ & $220(19,9)$ & $299(21,2)$ \\
\hline $16-17$ & $1.413(56,1)$ & $610(55,2)$ & $803(56,9)$ \\
\hline 18-19 & $585(23,2)$ & $276(25,0)$ & $309(21,9)$ \\
\hline \multicolumn{4}{|l|}{ Turno } \\
\hline Matutino & $723(28,7)$ & $325(29,4)$ & $398(28,2)$ \\
\hline Vespertino & $1.317(52,3)$ & $551(49,8)$ & $766(54,3)$ \\
\hline Noturno & $577(19,0)$ & $230(20,8)$ & $247(17,5)$ \\
\hline \multicolumn{4}{|l|}{ Renda Familiar (salários) } \\
\hline Até dois & $1.612(64,0)$ & $662(59,9)$ & $950(67,3)$ \\
\hline De três a cinco & $710(28,2)$ & $333(30,1)$ & $377(26,7)$ \\
\hline Seis ou mais & $195(7,7)$ & $111(10,0)$ & $84(6,0)$ \\
\hline \multicolumn{4}{|l|}{ Situação ocupacional } \\
\hline Trabalha & $456(18,1)$ & $252(22,8)$ & $204(14,5)$ \\
\hline Não trabalha & $2.061(81,9)$ & $854(77,2)$ & $1.207(85,5)$ \\
\hline \multicolumn{4}{|l|}{ Atividade Física } \\
\hline Inativo & $536(21,3)$ & $156(14,1)$ & $380(26,9)$ \\
\hline Insuficientemente ativo & $1.805(71,7)$ & $836(75,6)$ & $969(68,7)$ \\
\hline Ativo fisicamente & $176(7,0)$ & $114(10,3)$ & $62(4,4)$ \\
\hline
\end{tabular}

n: frequência absoluta; \%: frequência relativa. Inativo: zero minuto/dia; insuficientemente ativo: < 60 minutos/dia; Ativo fisicamente: $\geq 60$ minutos/dia.

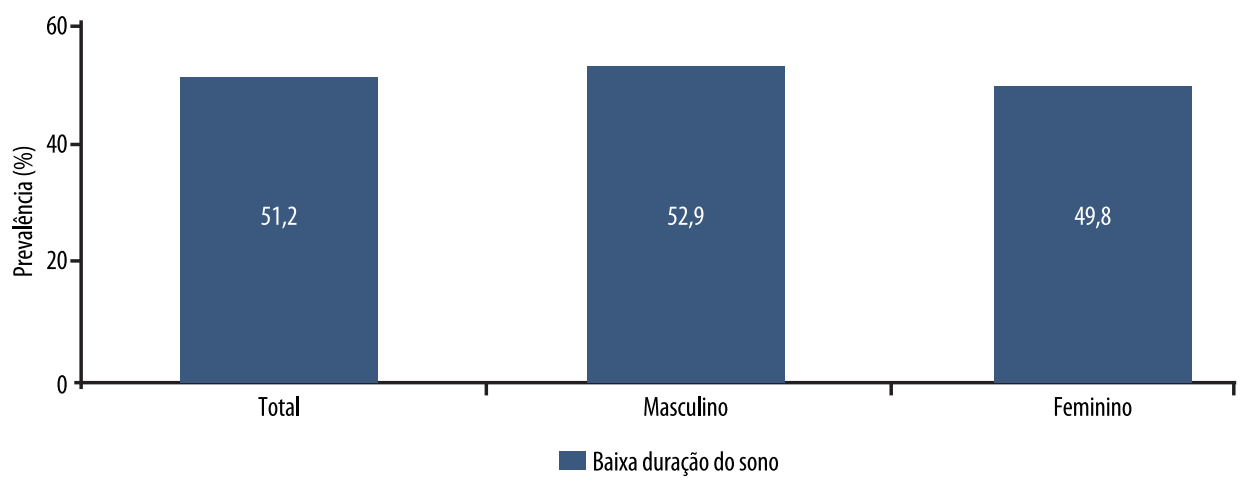

FIGURA 1 - Prevalência de baixa duração do sono em adolescentes do ensino médio do Amazonas, $2011(n=2.517)$.

$\mathrm{Na}$ análise bruta para identificação dos fatores associados à baixa duração do sono foi verificado que todas as variáveis se associaram a baixa duração do sono com exceção do sexo e renda familiar. Quando todas as variáveis foram consideradas no modelo ajustado as mesmas associações se mantiveram, indicando que os adolescentes mais velhos (18-19 anos) têm maior probabilidade ( $\mathrm{RP}=1,14$; IC95\%: 1,01-1,29) de apresentarem baixa duração do sono comparados aqueles mais jovens (14-15). Os adolescentes que estudam no turno matutino apresentam maior probabilidade ( $\mathrm{RP}=1,37$; IC95\%: 1,25-1,48) de terem baixa duração do sono, seguidos por aqueles do turno noturno ( $\mathrm{RP}=1,16$; IC95\%: 1,04-1,28) em relação aos seus pares do turno vespertino. Maiores probabilidades de terem baixa duração do sono foram observadas entre os adolescentes que trabalham (RP=1,34; IC95\%: 
1,23-1,45), bem como entre os que são inativos fisicamente ( $\mathrm{RP}=1,27$; IC95\%: 1,061,53) comparados com seus respectivos pares que não trabalhavam e que eram ativos fisicamente, respectivamente (Tabela 2).

TABELA 2 - Fatores associados com a baixa duração de sono nos adolescentes de escolas públicas do Amazonas, 2011 ( $n=2.517)$.

\begin{tabular}{|c|c|c|c|}
\hline \multirow[b]{2}{*}{ Variáveis } & \multirow{2}{*}{$\begin{array}{c}\text { Prevalência } \\
\text { n (\%) }\end{array}$} & \multicolumn{2}{|c|}{ Baixa duração do sono } \\
\hline & & $\begin{array}{c}\text { Bruta } \\
\text { RP (IC95\%) }\end{array}$ & $\begin{array}{c}\text { Ajustada } \\
\operatorname{RP}(\text { IC95\%) }\end{array}$ \\
\hline \multicolumn{4}{|l|}{ Sexo } \\
\hline Feminino & $703(49,8)$ & 1 & 1 \\
\hline Masculino & $585(52,9)$ & $1,06(0,98-1,15)$ & $1,05(0,97-1,13)$ \\
\hline \multicolumn{4}{|l|}{ Faixa etária (anos) } \\
\hline $18-19$ & $324(55,4)$ & $1,21(1,07-1,37)$ & $1,14(1,01-1,29)$ \\
\hline $16-17$ & $727(51,5)$ & $1,12(1,01-1,25)$ & $1,11(0,99-1,23)$ \\
\hline $14-15$ & $237(47,5)$ & 1 & 1 \\
\hline \multicolumn{4}{|l|}{ Turno } \\
\hline Noturno & $265(55,5)$ & $1,25(1,13-1,38)$ & $1,16(1,04-1,28)$ \\
\hline Matutino & $438(60,6)$ & $1,36(1,25-1,48)$ & $1,37(1,25-1,48)$ \\
\hline Vespertino & $585(44,4)$ & 1 & 1 \\
\hline \multicolumn{4}{|l|}{ Renda Familiar (salários) } \\
\hline Até dois & $844(52,4)$ & $1,01(0,88-1,17)$ & $1,08(0,94-1,24)$ \\
\hline De três a cinco & $343(48,3)$ & $0,93(0,80-1,09)$ & $0,97(0,83-1,49)$ \\
\hline Seis ou mais & $101(51,8)$ & 1 & 1 \\
\hline \multicolumn{4}{|l|}{ Situação ocupacional } \\
\hline Trabalha & $300(65,8)$ & $1,37(1,27-1,49)$ & $1,34(1,23-1,45)$ \\
\hline Não trabalha & $988(47,9)$ & 1 & 1 \\
\hline \multicolumn{4}{|l|}{ Atividade Física } \\
\hline Inativo fisicamente & $291(54,3)$ & $1,23(1,02-1,47)$ & $1,27(1,06-1,53)$ \\
\hline Insuficientemente ativo & $919(50,9)$ & $1,15(0,97-1,36)$ & $1,37(0,99-1,89)$ \\
\hline Ativo fisicamente & $78(44,3)$ & 1 & 1 \\
\hline
\end{tabular}

n: frequência absoluta; \%: frequência relativa; RP: razão de prevalência; IC95\%: intervalo de confiança de 95\%; Inativo: zero minuto/dia; insuficientemente ativo: < 60 minutos/dia; Ativo fisicamente: $\geq 60$ minutos/dia. *Análise ajustada por todas as variáveis.

\section{Discussão}

Foi observada uma elevada prevalência de baixa duração do sono (51,2\%; IC95\%: 49,3-52,8), sem diferença entre os sexos. As principais variáveis que se mantiveram associadas à baixa duração do sono foram estar na fase final da adolescência, estudar nos turnos matutino e noturno, trabalhar e a inatividade física.

Adolescentes no final da adolescência apresentaram maior prevalência de baixa duração do sono comparando-se aos mais jovens. Esse resultado corrobora com estudos prévios conduzidos com jovens brasileiros ${ }^{4,7,9}$. A baixa duração do sono deve-se a fatores sociocomportamentais e maturacionais, que se acentuam no final da puberdade ${ }^{20}$. Estes resultados podem implicar em dificuldade de aprendizagem, pior desempenho escolar e aumento do risco de sonolência excessiva ${ }^{21}$.

Ainda, como fator de risco a baixa duração do sono, os turnos matutino e noturno são os que pior se enquadram no relógio biológico do adolescente, especialmente no final da puberdade, período mais propenso ao atraso na fase de sono ${ }^{5}$. Com isso, fatores intrínsecos e sociais, atrasam a hora de deitar, somados aos com- 
promissos matutinos, como aulas ou trabalho, adiantam a hora de acordar, tornam-se fatores de risco à baixa duração do sono ${ }^{22}$. Supõe-se que este conjunto de fatores estejam colaborando com as altas prevalências de baixa duração do sono encontradas dos adolescentes que não atingiram as recomendações atuais de pelo menos oito horas de sono da National Sleep Foundation ${ }^{18}$.

Além da relação do relógio biológico versus relógio social, o trabalho esteve associado à baixa duração do sono. Uma revisão sistemática ${ }^{23} \mathrm{com}$ trabalhadores em geral apontou que demandas elevadas como o estresse no trabalho, assédio moral e desequilíbrio na questão esforço-recompensa estão relacionadas com distúrbios no sono. Esses fatores psicossociais e de programação trabalhista devem ser revistos de forma que auxiliem a minimizar o seu impacto nos problemas de sono dos adolescentes trabalhadores, pois na atual pesquisa os trabalhadores apresentaram duas vezes mais chances de apresentarem baixa duração do sono.

Durante o final da adolescência e o início da fase adulta existe um declínio na prática de $\mathrm{AF}^{1,2}$, mas não há consenso sobre seus efeitos na duração do sono. Estudo de revisão de Back et al. ${ }^{24}$ apontou a AF aeróbica como sincronizador não-fótico, que afeta o sistema de temporização circadiana (STC) por meio de vias não-fóticas, assim podendo adiantar ou atrasar o sono, desta forma, adequando os horários de sono, evitando privação.

Ainda, o exercício físico proporciona maiores gastos energéticos e exige maior reparação fisiológica e de energia em comparação ao indivíduo inativo fisicamente, logo reforça a necessidade de mais sono para restabelecer a homeostase perturbada pelo exercício físico ${ }^{25}$. Sendo assim, a prática regular de exercícios físicos pode acarretar em melhorias na eficiência e da qualidade do $\operatorname{sono}^{26}$, o que pode aumentar a duração do sono.

Além disso, outras explicações são possíveis, como a prática regular de AF poderia ter melhorado indiretamente o sono através de melhora do funcionamento psicológico, prevenindo o estresse e a ansiedade ${ }^{27,28}$. Contudo, as variáveis acerca do funcionamento psicológico não foram mensuradas, com isso considera-se isso uma suposição.

Ressalta-se que a prescrição da $\mathrm{AF}$ é recomendada como um tratamento não-farmacológico de distúrbios de sono ${ }^{25}$. Corroborando a ideia de que a $\mathrm{AF}$ tem ações positivas ao sono, os achados da presente pesquisa revelaram que os indivíduos inativos apresentam $61 \%$ mais chances de baixa duração do sono comparando-se aos adolescentes que alcançam os valores recomendados pela World Health Organization ${ }^{19}$.

Como pontos positivos desse estudo, destaca-se a amostra representativa do estado do Amazonas, bem como a adição das associações com o sono pouco investigado na literatura nacional. Já as principais limitações são o uso de questionário para avaliar as questões, que pode subestimar ou superestimar o tempo de sono e a AF. A utilização do ponto de corte para a duração do sono deve ser considerada como diretriz, pois não representa necessariamente o sono ideal de todos os indivíduos, apesar disso, é extremamente importante para o entendimento do fenômeno privação do sono. Ainda, o delineamento transversal que não possibilita estabelecer relação de causa e efeito.

Foram identificadas elevadas prevalências de baixa duração de sono nos adolescentes. Os fatores associados a oito ou menos horas de sono no grupo foram à fase final da adolescência (adolescentes mais velhos), os turnos matutino e noturno, trabalhar e a inatividade física. Sendo assim, fatores biológicos, comportamentais e ambientais são importantes para a duração do sono dos adolescentes.

Os adolescentes necessitam ser informados sobre a importância de uma boa 
higiene do sono, tanto quanto a prática de AF, devendo ser alertados dos decorrentes da baixa duração sono e do comportamento sedentário em demasia. Por fim, recomenda-se a organização de oportunidades de práticas de AF nas rotinas diária dos adolescentes, pois a AF constitui-se como elemento não-farmacológico para o tratamento em casos de baixa duração do sono, também como a adequação dos horários de sono com o horário de estudo para minimizar os agravos à saúde.

\section{Contribuição dos autores}

R. M. S. Puba Barbosa (0000-0003-1770-7777) e M. V. Nahas (0000-0002-43784031) contribuíram na elaboração do projeto de pesquisa e revisão crítica do manuscrito. A. A. Pinto (0000-0002-7931-3987) e G. J. Ferrari Junior (0000-00029739-3220) contribuíram na redação e revisão crítica do manuscrito. A. Pelegrini (0000-0001-8862-9636) e E. P. G. Felden (0000-0002-6924-122X) idealizaram o trabalho, realizaram as análises estatísticas, interpretação dos dados e revisão crítica do manuscrito. Os autores declaram que não nenhum conflito de interesses

\section{Conflito de interesses}

Não houve conflito de interesses.

\section{Financiamentos}

Não houve.

\section{Referências}

1. Caspersen CJ, Pereira MA, Curran KM. Changes in physical activity patterns in the United States, by sex and cross-sectional age. Med Sci Sports Exerc. 2000;32:1601-9.

2. Van Mechelen W, Twisk JW, Post GB, Snel J, Kemper HC. Physical activity of young people: the Amsterdam Longitudinal Growth and Health Study. Med Sci Sports Exerc. 2000;32:1610-6.

3. Fischer FM, Oliveira DC, Teixeira LR, Teixeira MCTV, Amaral MD. Efeitos do trabalho sobre a saúde de adolescentes. Cien Saúde Coletiva. 2013; 8(Suppl 4): 973-84.

4. Felden ÉPG, Filipin D, Barbosa DG, Andrade RD, Meyer C, Louzada FM. Fatores associados à baixa duração do sono em adolescentes. Rev Paul Pediatr. 2016; 34:64-70.

5. Crowley SJ, Acebo C, Carskadon MA. Sleep, circadian rhythms, and delayed phase in adolescence. Sleep Med. 2007;8:602-12.

6. Carskadon MA, Vieira C, Acebo C. Association between puberty and delayed phase preference sleep. Sleep. 1993;16:258-62.

7. Felden EPG, Ferrari Junior GJ, Andrade RD, Claumann GS, Pelegrini A, Teixeira CS. Fatores associados à baixa duração do sono em universitários ingressantes. R Bras Ci e Mov. 2015; 23(Suppl 4): 94-103.

8. Pereira EF, Bernardo MPSL, D'almeida V, Louzada FM. Sono, trabalho e estudo: duração do sono em estudantes trabalhadores e não trabalhadores. Cad Saúde Pública. 2011;27:975-84.

9. Bernardo MPSL, Pereira ÉF, Louzada FM, D’almeida V. Duração do sono em adolescentes de diferentes níveis socioeconômicos. J Bras Psiquiatr. 2009;58:231-37.

10. Pereira ÉF. Sono e sonolência diurna em adolescentes do ensino médio [Tese de doutorado]. Paraná: Universidade Federal do Paraná; 2012.

11. Legnani RFS, Legnani E, Gasparotto GS, Bacil EDA, Silva MP, Campos W. Hábitos de sono e atividade física em crianças e adolescentes: uma revisão sistemática. J Phys Educ. 2015. 26(1):147-56.

12. Javaheri S, Storfer-Isser A, Rosen CL, Redline S. Sleep quality and elevated blood pressure in adolescents. Circulation. 2008;118 (Suppl 10):1034-40.

13. Martins PJF, Mello MTD, Tufik S. Exercício e sono. Rev Bras Med Esporte. 2001; 7:28-36. 
14. Kreutzmann JC, Havekes R, Abel T, Meerlo P. Sleep deprivation and hippocampal vulnerability: changes in neuronal plasticity, neurogenesis and cognitive function. J Neurosci. 2015; 309:173-90.

15. Kredlow MA, Capozzoli MC, Hearon BA, Calkins AW, Otto MW. The effects of physical activity on sleep: a meta-analytic review. J Behav Med. 2015;38:427-49.

16. Luiz RR, Magnanini MMF. O tamanho da amostra em investigações epidemiológicas. Cad Saúde Colet. 2000;8( Suppl 2):9-28.

17. Silva KS, Lopes AS, Hoefelmann LP, Cabral LG, De Bem MF, Barros MV, et al. Projeto Compac (Comportamento dos Adolescentes Catarinenses): aspectos metodológicos, operacionais e éticos. Rev Bras Cineantropom Desempenho Hum. 2013;15( Suppl 1):1-15.

18. Hirshkowitz M, Whiton K, Albert SM, Alessi C, Bruni O, DonCarlos L, et al. National Sleep Foundation's sleep time duration recommendations: methodology and results summary. Sleep Health. 2015;1:40-3.

19. World Health Organization. Global recommendations on physical activity for health. Geneva: WHO; 2010. Acesso em 21 de outubro de 2016. Disponível em: http://www.who. int/dietphysicalactivity/factsheet_recommendations/en/

20. Moore M, Meltzer LJ. The sleepy adolescent: causes and consequences of sleepiness in teens. Paediatr Respir Rev. 2008;9:114-21.

21. Carskadon MA, Acebo C, Jenni OG. Regulation of adolescent sleep: implications for behavior. Ann N Y Acad Sci. 2004;1021:276-91.

22. Wolfson AR, Carskadon MA. Sleep schedules and daytime functioning in adolescents. Child Dev. 1998;69:875-87.

23. Linton SJ, Kecklund G, Franklin KA, Leissner LC, Sivertsen B, Lindberg E, et al. The effect of the work environment on future sleep disturbances: a systematic review. Sleep Med Rev. 2015;23:10-19.

24. Back FL, Fortes FS, Santos EHR, Tambelli R, Menna-Barreto LS, Louzada FM. Sincronização não-fótica: o efeito do exercício físico aeróbio. Rev Bras Med Esporte. 2007; 13(Suppl 2):1-5.

25. Brand S, Gerber M, Beck J, Hatzinger M, Pühse U, Holsboer-Trachsler E. High exercise levels are related to favorable sleep patterns and psychological functioning in adolescents: a comparison of athletes and controls. J Adolesc Health. 2010 Feb;46(2):133-41

26. Chennaoui M, Arnal PJ, Sauvet F, Léger D. Sleep and exercise: a reciprocal issue?. Sleep Med Rev. 2015;20:59-72

27. Driver HS, Taylor S. Exercise and sleep. Sleep Med Rev 2000;4:387-402

28. Biddle SJ, Fox KR. Exercise and health psychology: Emerging relationships. British J Med Psychol. 1989, 62(Suppl 3), 205-16.

29. Dunn AL, Trivedi MH, Kampert JB, Clark CG, Chambliss HO. Exercise treatment for depression: efficacy and dose response. Am J Prev Med. 2005;28( Suppl 1):1-8.

Endereço para Correspondência

Érico Gomes Pereira Felden

ericofelden@gmail.com
Grupo de Pesquisa Atividade

Física e Sono

Rua Pascoal Simone, 358, Coqueiros,

Florianópolis - SC. CEP: 88080-350

Telefone: (48) 3664-8695
Recebido

Revisado

$13 / 12 / 2016$ 28/04/2017 $30 / 04 / 2017$ 